\title{
A simplified model for mitochondrial ATP production
}

\author{
Richard Bertram $^{\mathrm{a}, *}$, Morten Gram Pedersen ${ }^{\mathrm{b}}$, Dan S. Luciani ${ }^{\mathrm{c}}$, Arthur Sherman ${ }^{\mathrm{d}}$ \\ ${ }^{a}$ Department of Mathematics and Programs in Neuroscience and Molecular Biophysics, Florida State University, Tallahassee, FL 32306, USA \\ ${ }^{\mathrm{b}}$ Department of Mathematics, Technical University of Denmark, Kgs. Lyngby, Denmark \\ ${ }^{\mathrm{c}}$ Department of Cellular and Physiological Sciences, University of British Columbia, Vancouver, Canada \\ ${ }^{\mathrm{d}}$ Laboratory of Biological Modeling, National Institutes of Health, Bethesda, MA, USA
}

Received 17 May 2006; received in revised form 4 July 2006; accepted 20 July 2006

Available online 25 July 2006

\begin{abstract}
Most of the adenosine triphosphate (ATP) synthesized during glucose metabolism is produced in the mitochondria through oxidative phosphorylation. This is a complex reaction powered by the proton gradient across the mitochondrial inner membrane, which is generated by mitochondrial respiration. A detailed model of this reaction, which includes dynamic equations for the key mitochondrial variables, was developed earlier by Magnus and Keizer. However, this model is extraordinarily complicated. We develop a simpler model that captures the behavior of the original model but is easier to use and to understand. We then use it to investigate the mitochondrial responses to glycolytic and calcium input. We use the model to explain experimental observations of the opposite effects of raising cytosolic $\mathrm{Ca}^{2+}$ in low and high glucose, and to predict the effects of a mutation in the mitochondrial enzyme nicotinamide nucleotide transhydrogenase $(\mathrm{Nnt})$ in pancreatic $\beta$-cells.
\end{abstract}

(C) 2006 Elsevier Ltd. All rights reserved.

Keywords: Metabolism; Calcium; Mathematical model

\section{Introduction}

The mitochondria are the primary location for the production of energy-carrying molecules in most cells. Metabolism begins in the cytoplasm with glycolysis, where glucose is metabolized to the primary product pyruvate. The pyruvate enters the mitochondria through shuttles, where it is processed by the citric acid cycle. The coenzymes nicotinamide adenine dinucleotide $\left(\mathrm{NAD}^{+}\right)$and flavin adenine dinucleotide (FAD) are reduced during the citric acid cycle, yielding NADH and $\mathrm{FADH}_{2}$ (some NADH is also generated during glycolysis). These electron carriers are used by the electron transport chain (ETC), which supplies the energy to establish a proton gradient across the inner membrane of the mitochondria. The gradient is used to power the last stage of metabolism, oxidative phosphorylation. Protons flowing down this gradient through the ATP synthase complex provide the energy to phosphorylate adenosine diphosphate (ADP) to adenosine tripho-

\footnotetext{
${ }^{*}$ Corresponding author. Tel.: 8506447195 ; fax: 8506444053

E-mail address: bertram@math.fsu.edu (R. Bertram).
}

sphate (ATP). This is the primary means through which the energy molecule ATP is produced in most eukaryotic cells.

In a series of papers in the late 1990s, Magnus and Keizer developed mathematical models of oxidative phosphorylation (Magnus and Keizer, 1997, 1998a, 1998b). These models were developed in the context of the insulin-secreting pancreatic $\beta$-cell, and were coupled to models of the cell's electrical activity and cytosolic $\mathrm{Ca}^{2+}$ handling. The Magnus-Keizer $(\mathrm{M}-\mathrm{K})$ model includes most of the processes thought to be important for mitochondrial oxidative phosphorylation, and indeed, the completeness of the $\mathrm{M}-\mathrm{K}$ model is one of its strengths. The model was later used and modified by Cortassa et al., 2003 to describe metabolism in cardiac cells. The Cortassa model also makes some improvements to the $\mathrm{M}-\mathrm{K}$ model and includes a model, which we do not use here, for the citric acid cycle developed by Dudycha and Jafri (Dudycha, 2000; Jafri et al., 2001).

The $\mathrm{M}-\mathrm{K}$ model was derived from first principles, and as a result it is very complex. Many of the properties of the model, such as whether a flux term increases or decreases when the value of a variable or parameter is changed, are 
masked by this complexity. Our first goal is to develop a simplified model for oxidative phosphorylation that retains the key features of the $\mathrm{M}-\mathrm{K}$ model, yet is more intuitive. A description of this simplified model, and comparison with the $\mathrm{M}-\mathrm{K}$ model as modified by Cortassa et al., is the focus of the first portion of the article.

In the second portion, we use the simplified model to study the response of the mitochondrial variables to pulses of calcium and a variable reflecting glycolytic flux, both of which are inputs to mitochondrial metabolism. This yields predictions about whether the mitochondrial variables increase or decrease in response to the input. Measurements have been made of the levels of $\mathrm{NAD}(\mathrm{P}) \mathrm{H}$, oxygen, and the mitochondrial inner membrane potential in, for example, pancreatic $\beta$-cells. Besides the clinical significance of these insulin-secreting cells, they are interesting since glucose metabolism is important not only for the survival of the $\beta$-cell, but also for its electrical activity and patterned insulin release.

We next use the model to investigate a recent experimental result from mouse pancreatic islets that has been hard to explain. It was found that elevations in the intracellular $\mathrm{Ca}^{2+}$ concentration increase the intracellular NADH concentration when the islet is maintained in a low glucose bath. In higher glucose, a $\mathrm{Ca}^{2+}$ concentration increase results in a reduction in the NADH concentration (Luciani et al., 2006). Our model provides a plausible mechanism for this.

Finally, we consider the effects of a negative mutation in the mitochondrial enzyme nicotinamide nucleotide transhydrogenase (Nnt). Such a mutation causes glucose intolerance and impaired $\beta$-cell function in the widely used C57BL/6J mouse strain (Toye et al., 2005). In the simplified model, simulation of the Nnt mutation is accomplished by increasing the proton leak across the mitochondrial inner membrane. Increase of this single parameter results in changes in the mitochondrial variables that would not be obvious without a mathematical model. This illustrates one of the strengths of the simplified model: its relative simplicity makes it possible to predict and understand the effects of changes in one or more parameters or variables. This is particularly important when the mitochondrial model is just one component of a larger cellular model.

\section{Dynamic equations}

We begin by describing the dynamic equations for the mitochondrial variables $N A D H_{m}, A D P_{m}, \Delta \Psi$, and $C a_{m}$. These equations are themselves simple. However, the flux and reaction terms that make up the equations (Fig. 1) are quite complex in the $\mathrm{M}-\mathrm{K}$ model, and it is those that we simplify in the following section.

The first stage of glucose metabolism in eukaryotic cells is glycolysis, which takes place in the cytoplasm. Although this produces some ATP and NADH, its primary output is pyruvate. The pyruvate is transported into the mitochondria where it is rapidly oxidized and decarboxylated by the

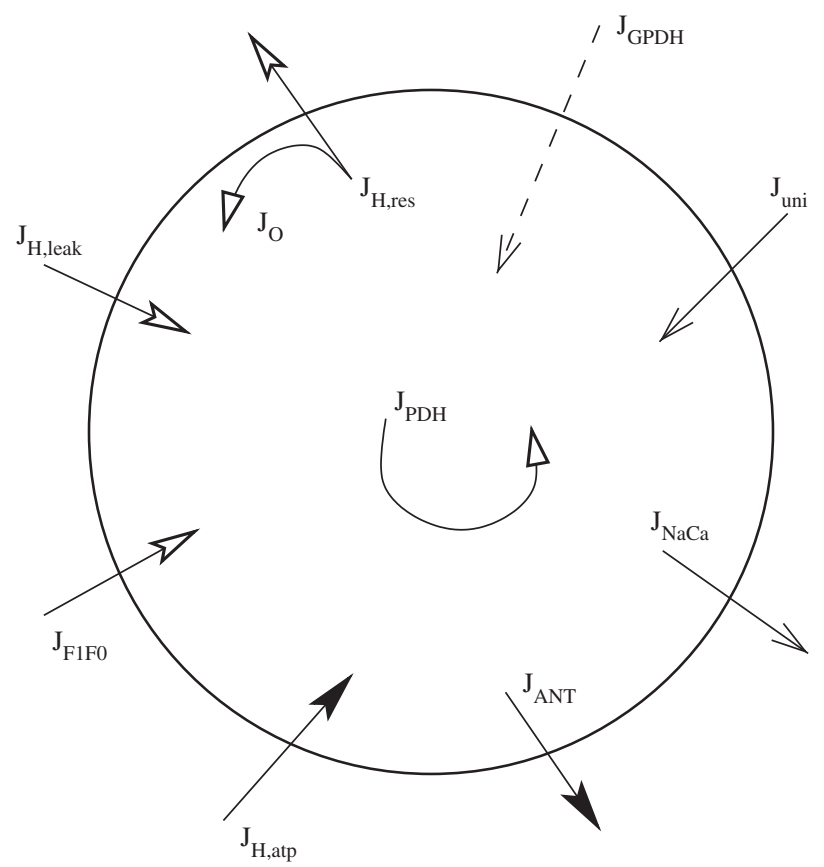

Fig. 1. Illustration of the fluxes and reactions used in the model. The different arrow types correspond to different types of fluxes or reactions. Arrows with line-type arrow heads represent the flux of $\mathrm{Ca}^{2+}$. Arrows with open arrow heads represent proton fluxes. Arrows with closed arrow heads represent nucleotide fluxes or phosphorylation events. Curved arrows represent the production or oxidation of NADH. Finally, the dashed arrow represents input to the mitochondria from glycolysis.

pyruvate dehydrogenase complex $(\mathrm{PDH})$. The products of PDH are a molecule of $\mathrm{CO}_{2}$, a molecule of NADH, and acetyl coenzyme A (acetyl CoA). The acetyl CoA enters the citric acid cycle, where more NADH is produced by additional dehydrogenases. As was done by Magnus and Keizer, we assume that the citric acid dehydrogenase rates are proportional to the reaction rate of $\mathrm{PDH}$, and let $J_{P D H}$ represent the reaction rate for the sum of the dehydrogenases. The NADH concentration is decreased by the action of the ETC, during which NADH is converted to $\mathrm{NAD}^{+}$and oxygen is consumed:

$\frac{\mathrm{d} N A D H_{m}}{\mathrm{~d} t}=\gamma\left(J_{P D H}-J_{o}\right)$,

where $N A D H_{m}$ is the mitochondrial NADH concentration, and $J_{o}$ is the oxygen consumption rate. Both $J_{P D H}$ and $J_{o}$ have units of $\mu \mathrm{M} / \mathrm{ms}$. The time $t$ is measured in $\mathrm{ms}$, and $\gamma=0.001$ converts $N A D H_{m}$ to units of $\mathrm{mM}$.

The mitochondrial ADP concentration increases due to the action of the adenine nucleotide transporter, which transports ATP out of and ADP into the mitochondria. ADP concentration decreases due to the action of the ATP synthase, which phosphorylates ADP to ATP. Thus,

$\frac{\mathrm{d} A D P_{m}}{\mathrm{~d} t}=\gamma\left(J_{A N T}-J_{F 1 F 0}\right)$,

where $A D P_{m}$ is the mitochondrial ADP concentration (with units of $\mathrm{mM}$ ), $J_{A N T}$ is the nucleotide transport rate, and $J_{F 1 F 0}$ is the ATP synthase rate (both in $\mu \mathrm{M} / \mathrm{ms}$ ). 
Table 1

Parameter Values for the Simplified Model

\begin{tabular}{|c|c|c|}
\hline$p_{1}=400$ & $p_{2}=1$ & $p_{3}=0.01 \mu \mathrm{M}$ \\
\hline$p_{4}=0.6 \mu \mathrm{Mms}^{-1}$ & $p_{5}=0.1 \mathrm{mM}$ & $p_{6}=177 \mathrm{mV}$ \\
\hline$p_{7}=5 \mathrm{mV}$ & $p_{8}=7 \mu \mathrm{Mms}^{-1}$ & $p_{9}=0.1 \mathrm{mM}$ \\
\hline$p_{10}=177 \mathrm{mV}$ & $p_{11}=5 \mathrm{mV}$ & $p_{12}=120 \mu \mathrm{Mms}^{-1}$ \\
\hline$p_{13}=10 \mathrm{mM}$ & $p_{14}=190 \mathrm{mV}$ & $p_{15}=8.5 \mathrm{mV}$ \\
\hline$p_{16}=35 \mu \mathrm{Mms}^{-1}$ & $p_{17}=0.002 \mu \mathrm{Mms}^{-1} \mathrm{mV}^{-1}$ & $p_{18}=-0.03 \mu \mathrm{Mms}^{-1}$ \\
\hline$p_{19}=0.35 \mu \mathrm{Mms}^{-1}$ & $p_{20}=2$ & $\mathrm{FRT}=0.037 \mathrm{mV}^{-1}$ \\
\hline$p_{21}=0.01 \mu \mathrm{M}^{-1} \mathrm{~ms}^{-1} \mathrm{mV}^{-1}$ & $p_{22}=1.1 \mu \mathrm{M}^{-1} \mathrm{~ms}^{-1}$ & $p_{23}=0.001 \mu \mathrm{Mms}^{-1}$ \\
\hline$p_{24}=0.016 \mathrm{mV}^{-1}$ & $N A D_{t o t}=10 \mathrm{mM}$ & $A_{t o t}=15 \mathrm{mM}$ \\
\hline$C_{m}=1.8 \mu \mathrm{M} \mathrm{mV}^{-1}$ & $f_{m}=0.01$ & $k_{G P D H}=5 \times 10^{-4} \mu \mathrm{Mms}^{-1}$ \\
\hline
\end{tabular}

We assume that the mitochondrial concentrations of adenine and pyridine nucleotides are conserved:

$N A D_{m}+N A D H_{m}=N A D_{t o t}$,

$A D P_{m}+A T P_{m}=A_{\text {tot }}$,

where $N A D_{\text {tot }}$ and $A_{\text {tot }}$ are the total concentrations (in $\mathrm{mM}$, see Table 1).

The inner membrane potential, $\Delta \Psi$, is influenced by many fluxes and reactions:

$$
\begin{aligned}
\frac{\mathrm{d} \Delta \Psi}{\mathrm{d} t}= & \left(J_{H, r e s}-J_{H, a t p}-J_{A N T}-J_{H, l e a k}\right. \\
& \left.-J_{N a C a}-2 J_{u n i}\right) / C_{m},
\end{aligned}
$$

where $J_{H, \text { res }}$ is the respiration-driven proton flux, $J_{H, \text { atp }}$ is the proton flux through the ATP synthase, $J_{H, \text { leak }}$ is the proton leak across the mitochondrial inner membrane, $J_{\mathrm{NaCa}}$ is the $\mathrm{Ca}^{2+}$ efflux through the electrogenic $\mathrm{Na}^{+} / \mathrm{Ca}^{2+}$ exchanger, and $J_{u n i}$ is the $\mathrm{Ca}^{2+}$ influx through the $\mathrm{Ca}^{2+}$ uniporter. The mitochondrial capacitance is $C_{m}$, and $\Delta \Psi$ has units of $\mathrm{mV}$.

Finally, the $\mathrm{Ca}^{2+}$ concentration in the mitochondria $\left(C a_{m}\right)$ is increased by the $\mathrm{Ca}^{2+}$ uniporter and decreased by the $\mathrm{Na} / \mathrm{Ca}$ exchanger:

$\frac{\mathrm{d} C a_{m}}{\mathrm{~d} t}=f_{m}\left(J_{u n i}-J_{N a C a}\right)$,

where $f_{m}$ is the fraction of free $\mathrm{Ca}^{2+}$.

Rather than using pyruvate concentration as input to $\mathrm{PDH}$, the glyceraldehyde 3-phosphate dehydrogenase (GPDH) activity is used in our description. This is a glycolytic enzyme downstream of the key allosteric enzyme phosphofructokinase (PFK) in the glycolytic pathway. Thus, the GPDH reaction rate reflects the flux through the glycolytic pathway. We assume that the GPDH reaction is in rapid equilibrium. Tornheim (1979) then argued that it can be described approximately by an algebraic function of the substrate fructose 1,6-bisphosphate (FBP),

$J_{G P D H}=k_{G P D H} \sqrt{(F B P) /(1 \mu \mathrm{M})}$,

where FBP has units of $\mu \mathrm{M}$ and $J_{G P D H}$ has units of $\mu \mathrm{Mms}^{-1}$. The cytosolic $\mathrm{Ca}^{2+}$ concentration, $C a_{c}$, is another input to mitochondrial respiration, since cytosolic $\mathrm{Ca}^{2+}$ enters the mitochondria through $\mathrm{Ca}^{2+}$ uniporters,

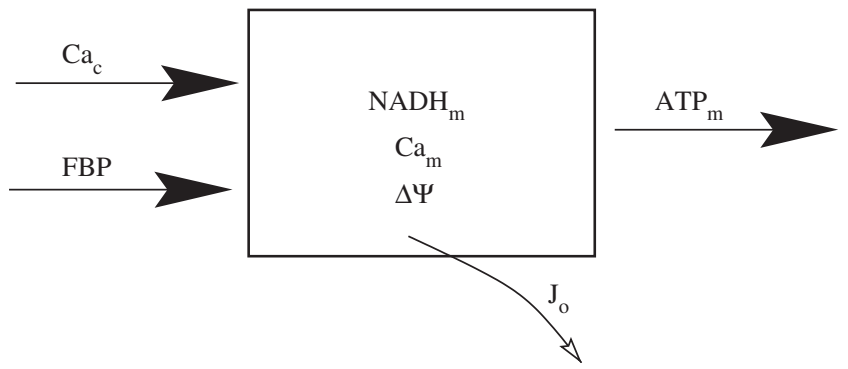

Fig. 2. Input and output of mitochondrial respiration, and mitochondrial variables present in the model. Oxygen consumption, $J_{o}$, is also illustrated since it is an observable quantity.

and affects the mitochondrial dehydrogenases and the inner membrane potential.

The end product of metabolism is ATP. Thus, the output variable for mitochondrial metabolism is the mitochondrial ATP concentration. This is turn determines the cytosolic ATP concentration, through the action of the adenine nucleotide translocator. Input, output, and mitochondrial variables are illustrated in Fig. 2. Also illustrated is the oxygen flux, since this is a measurable quantity.

\section{Model simplification}

In this section we describe the flux and reaction rates used in the dynamic equations from the previous section. We begin the descriptions of the terms with mathematical expressions from Cortassa et al. (2003), which are themselves modifications of expressions from the $\mathrm{M}-\mathrm{K}$ model (Magnus and Keizer, 1998a, 1998b). The Cortassa expressions are used since they have been somewhat improved over those from the $\mathrm{M}-\mathrm{K}$ model. We still, however, refer to these as " $\mathrm{M}-\mathrm{K}$ expressions" or " $\mathrm{M}-\mathrm{K}$ functions" since the original formulation was by Magnus and Keizer.

We begin with the pyruvate dehydrogenase (PDH) reaction rate, $\hat{J}_{P D H}$. (The hat superscript is used here to distinguish the $\mathbf{M}-\mathrm{K}$ expression from our simplified expression.) This reaction decarboxylates pyruvate, but since we use GPDH as input to the mitochondria, we use the GPDH reaction rate (Eq. 7) as input to PDH. PDH is also known to be stimulated by mitochondrial $\mathrm{Ca}^{2+}$. We modify the $\mathrm{M}-\mathrm{K}$ expression for $\hat{J}_{P D H}$ by adding an explicit 
dependence on the ratio of product NADH to substrate $\mathrm{NAD}^{+}$. The modified expression for the $\mathrm{PDH}$ reaction rate (in units of $\mu \mathrm{M} / \mathrm{ms}$ ) is

$\hat{J}_{P D H}=\left(V_{P D H} F_{P D H} J_{G P D H}\right) / \gamma_{2}$,

where $V_{P D H}=77 \mu \mathrm{M} / \mathrm{s}, \quad \gamma_{2}=0.1$ is the mitochondria/ cytosol volume ratio, and $J_{G P D H}$ is the GPDH reaction rate. (Cortassa et al. converted $\mathrm{M}-\mathrm{K}$ parameter values to SI units. We use these parameter values, and convert from $\mathrm{mM}$ to $\mu \mathrm{M}$.) Finally,

$F_{P D H}=\frac{1}{1+u_{2}\left[\left(1+u_{1}\left(1+C a_{m} / K_{P D H c a}\right)^{-2}\right) /\left(N A D H_{m} / N A D_{m}+K_{P D H n a d}\right)\right]}$.

Here $u_{1}=1.5, u_{2}=1.0, K_{\text {PDHca }}=0.05 \mu \mathrm{M}$ and $K_{\text {PDHnad }}=$ 1. In the $\mathrm{M}-\mathrm{K}$ model it was assumed that the reaction rate of citric acid dehydrogenases is proportional to that of PDH (Magnus and Keizer, 1998a), and $\hat{J}_{P D H}$ represents the sum of these two.

The simplified PDH reaction rate function that we use is

$J_{P D H}=\left(\frac{p_{1}}{p_{2}+N A D H_{m} / N A D_{m}}\right)\left(\frac{C a_{m}}{p_{3}+C a_{m}}\right) J_{G P D H}$.

Values for the three parameters $\left(p_{1}, p_{2}, p_{3}\right)$, as well as other parameter values for the simplified model, are given in Table 1. The PDH reaction rate computed with the two models is shown in Fig. 3. The top panel shows that $J_{P D H}$ increases linearly with $J_{G P D H}$, and $J_{P D H}$ computed with Eq. (10) (dashed curve) closely matches that computed with Eq. (8) (solid curve). The dependence on NADH is shown in Fig. 3(B), with the total NAD concentration $\left(N A D H_{m}+N A D_{m}\right)$ held constant at $10 \mathrm{mM}$. The PDH rate decreases when $N A D H_{m}$ is increased (and $N A D_{m}$ decreased) since $\mathrm{NAD}^{+}$is a substrate for $\mathrm{PDH}$. The dependence of $J_{P D H}$ on the mitochondrial $\mathrm{Ca}^{2+}$ concentration is shown in Fig. 3(C). The dehydrogenase rate increases with $C a_{m}$, but saturates at relatively low values of $\mathrm{Ca}_{m}$. This $\mathrm{Ca}^{2+}$ dependence becomes important later, when we study the effects of input pulses on the mitochondrial variables.

Oxygen is consumed during the final stage of electron transport, and this reflects conversion of NADH to NAD ${ }^{+}$ by the loss of electrons. The expression for $\mathrm{O}_{2}$ consumption $\left(J_{o}\right)$ is quite complex in the M-K model, with dependency on $\mathrm{NAD}^{+}, \mathrm{NADH}$, and the proton motive force $\left(\Delta \mu_{H}\right)$ :

$\hat{J}_{o}=\frac{0.5 \rho_{\text {res }}(A-B+C)}{D}$,

where

$A=K_{r e s}\left(r_{a}+r_{c 1} \mathrm{e}^{6 \Delta \Psi_{B} F R T}\right) \sqrt{N A D H_{m} / N A D_{m}}$,

$B=r_{a} \mathrm{e}^{6 g \Delta \mu_{H} F R T}$,

$C=r_{c 2} K_{r e s} \mathrm{e}^{6 g \Delta \mu_{H} F R T} \sqrt{N A D H_{m} / N A D_{m}}$,
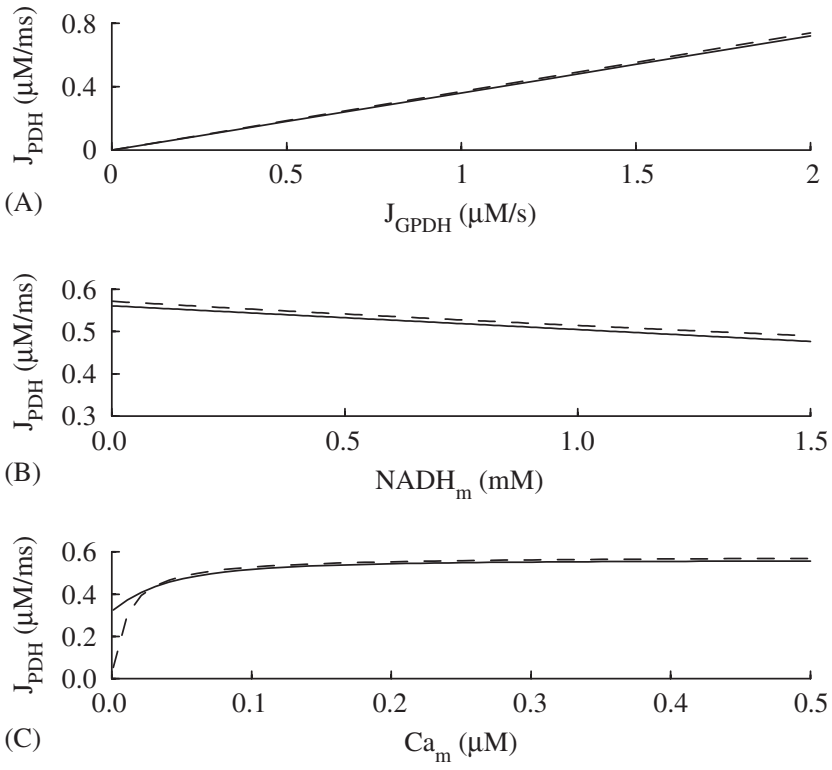

Fig. 3. PDH reaction rate computed with Eq. (8) (solid curves) and the simplified expression (Eq. (10), dashed curves). (A) $J_{P D H}$ increases linearly with $J_{G P D H}$. $\left(N A D H_{m}=0.3 \mathrm{mM}, C a_{m}=0.2 \mu \mathrm{M}\right.$.) (B) The reaction rate decreases when $N A D H_{m}$ is increased and $N A D_{m}$ is decreased, maintaining $N A D H_{m}+N A D_{m}=10 \mathrm{mM}\left(J_{G P D H}=1.5 \mu \mathrm{M} / \mathrm{s}, C a_{m}=0.2 \mu \mathrm{M}\right)$. (C) The dehydrogenase saturates at higher values of $\mathrm{Ca}_{m}\left(N A D H_{m}=0.3 \mathrm{mM}\right.$, $\left.J_{G P D H}=1.5 \mu \mathrm{M} / \mathrm{s}\right)$.

$$
\begin{aligned}
D= & \left(1+r_{1} K_{\text {res }} \sqrt{N A D H_{m} / N A D_{m}}\right) \mathrm{e}^{6 \Delta \Psi_{B} F R T} \\
& +\left(r_{2}+r_{3} K_{\text {res }} \sqrt{N A D H_{m} / N A D_{m}}\right) \mathrm{e}^{6 g \Delta \mu_{H} F R T} .
\end{aligned}
$$

Here $\rho_{\text {res }}=0.6036 \mu \mathrm{M}, \quad K_{\text {res }}=1.35 \times 10^{18}, \quad r_{a}=6.394 \times$ $10^{-13} \mathrm{~ms}^{-1}, \quad r_{c 1}=2.656 \times 10^{-22} \mathrm{~ms}^{-1}, \quad r_{c 2}=8.632 \times 10^{-30}$ $\mathrm{ms}^{-1}, \Delta \Psi_{B}=50 \mathrm{mV}, F R T=F / R T=0.037 \mathrm{mV}^{-1}$ is Faraday's constant divided by the gas constant and temperature, $g=0.85, r_{1}=2.077 \times 10^{-18}, r_{2}=1.728 \times 10^{-9}$, and $r_{3}=1.059 \times 10^{-26}$. See Magnus and Keizer (1998a) or Cortassa et al. (2003) for a description of parameters. The proton motive force, $\Delta \mu_{H}$, includes both the membrane potential, $\Delta \Psi$, and a contribution due to the proton concentration gradient, $\triangle p H$ :

$\Delta \mu_{H}=\Delta \Psi+\frac{\Delta p H}{F R T}$,

where $\Delta p H=-0.6$.

Our simplified expression for $J_{o}$ is

$J_{o}=\left(\frac{p_{4} N A D H_{m}}{p_{5}+N A D H_{m}}\right)\left(\frac{1}{1+\exp \left(\left(\Delta \Psi-p_{6}\right) / p_{7}\right)}\right)$.

Fig. 4 shows the $\mathrm{O}_{2}$ consumption rates calculated from Eqs. (11) and (17), with $p_{4}-p_{7}$ adjusted to match the $\mathrm{M}-\mathrm{K}$ curves. The consumption rate declines for large $\Delta \Psi$ (Fig. 4(A)), since it is more difficult to pump protons against a large potential (metabolic control). The consumption rate increases with the NADH concentration (Fig. 4(B)), since NADH is an electron donor.

As oxygen is consumed by the ETC, protons are pumped across the inner mitochondrial membrane. Thus, the respiration-driven proton flux, $J_{H, r e s}$, is linked to $\mathrm{O}_{2}$ 

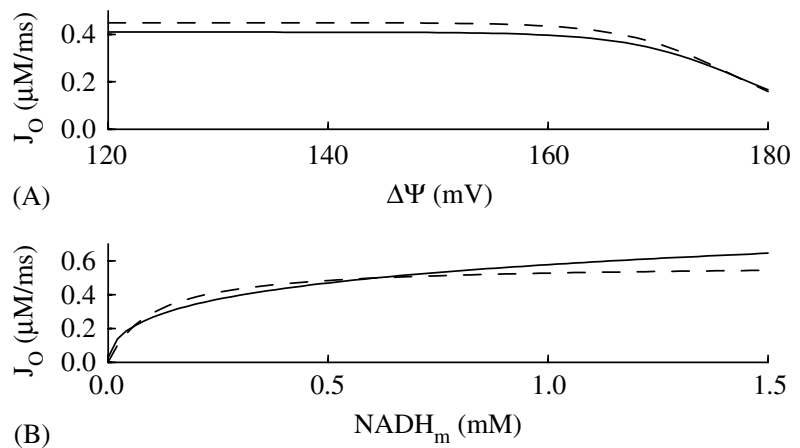

Fig. 4. Oxygen consumption rate, calculated with the $\mathrm{M}-\mathrm{K}$ expression (Eq. (11), solid curves) and with the simplified expression (Eq. (17), dashed curves). (A) $J_{o}$ declines with $\Delta \Psi$, reflecting metabolic control $\left(N A D H_{m}=0.3 \mathrm{mM}\right)$. (B) $J_{o}$ increases with $\mathrm{NADH}$, the electron donor to the electron transport chain $(\Delta \Psi=160 \mathrm{mV})$.

consumption. Both NADH and FADH are electron donors, but in the $\mathrm{M}-\mathrm{K}$ model $\mathrm{NADH}$ is the primary donor. We therefore omit the contribution to $J_{H \text {,res }}$ from FADH. The $\mathrm{M}-\mathrm{K}$ expression for the contribution to $J_{H, \text { res }}$ from $\mathrm{NADH}$ is:

$\hat{J}_{H, \text { res }}=\frac{6 \rho_{\text {res }}(E-F)}{D}$,

where

$E=r_{a} K_{r e s} \sqrt{N A D H_{m} / N A D_{m}}$,

$F=\left(r_{a}+r_{b}\right) \mathrm{e}^{\left(6 g \Delta \mu_{H} F R T\right)}$

and $D$ is given by Eq. (15). The simplified expression for $J_{H, \text { res }}$ is similar to that for $J_{o}$,

$J_{H, \text { res }}=\left(\frac{p_{8} N A D H_{m}}{p_{9}+N A D H_{m}}\right)\left(\frac{1}{1+\exp \left(\left(\Delta \Psi-p_{10}\right) / p_{11}\right)}\right)$.

Fig. 5 shows $J_{H, \text { res }}$ calculated from both Eqs. (18) and (21). As expected, the dependence of this flux term on $\Delta \Psi$ and $N A D H_{m}$ is similar to that of $J_{o}$ (Fig. 4).

ADP is converted to ATP using the energy provided by the flow of protons down their concentration gradient through the $\mathrm{F}_{1} \mathrm{~F}_{0}$ ATP-synthase. This is the step at which most of the ATP is formed during glucose metabolism. Proton flux through the ATP-synthase in the $\mathrm{M}-\mathrm{K}$ model is

$\hat{J}_{H, a t p}=-3 \rho^{F 1} \frac{G-H}{K+L}$,

where $\rho^{F 1}=1.787 \mathrm{mM}$ is the concentration of the ATPsynthase, and

$$
\begin{aligned}
& G=100 p_{a}\left(1+\mathrm{e}^{A_{F 1} F R T}\right), \\
& H=-\left(p_{a}+p_{b}\right) \mathrm{e}^{3 \Delta \mu_{H} F R T}, \\
& K=\left(1+q_{1} \mathrm{e}^{A_{F 1} F R T}\right) \mathrm{e}^{3 \Delta \Psi_{B} F R T}, \\
& L=\left(q_{2}+q_{3} \mathrm{e}^{A_{F 1} F R T}\right) \mathrm{e}^{3 \Delta \mu_{H} F R T},
\end{aligned}
$$
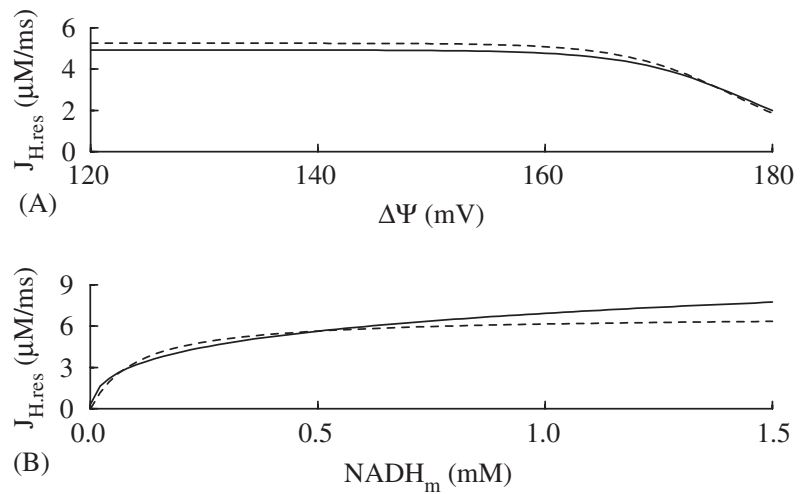

Fig. 5. Proton flux across the inner mitochondrial membrane due to respiration, calculated with the $\mathrm{M}-\mathrm{K}$ expression (Eq. (18)) and the simplified expression (Eq. (21)). (A) $J_{H, r e s}$ decreases with $\Delta \Psi$, reflecting metabolic control $\left(N A D H_{m}=0.3 \mathrm{mM}\right)$. (B) Proton flux increases with an increase in the concentration of the electron donor to the electron transport chain. $(\Delta \Psi=160 \mathrm{mV}$.)
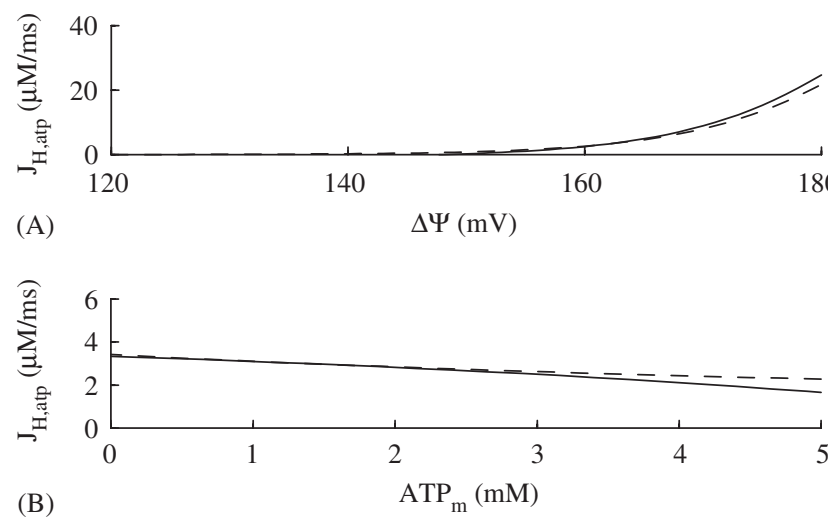

Fig. 6. Proton flux through the ATP-synthase, calculated with the M-K expression (Eq. (22), solid curves) and with the simplified expression (Eq. (28), dashed curves). (A) $J_{H, \text { atp }}$ increases with the electrical gradient, $\Delta \Psi$. $\left(A T P_{m}=3 \mathrm{mM}\right)$. (B) $J_{H, a t p}$ declines as the concentration of the reaction product increases. $(\Delta \Psi=160 \mathrm{mV})$

and where

$A_{F 1}=\ln \left(K_{F 1} \frac{A T P_{m}}{P_{i} A D P_{m}}\right) / F R T$.

Parameter values are $p_{a}=1.656 \times 10^{-8} \mathrm{~ms}^{-1}, p_{b}=3.373 \times$ $10^{-10} \mathrm{~ms}^{-1}, \quad q_{1}=1.346 \times 10^{-8}, \quad q_{2}=7.739 \times 10^{-7}, \quad q_{3}=$ $6.65 \times 10^{-15}, K_{F 1}=1.71 \times 10^{6}$, and $P_{i}=20 \mathrm{mM}$ is the inorganic phosphate concentration.

In the simplified expression for $J_{H, \text { atp }}$ there is an increasing sigmoidal dependence on $\Delta \Psi$ and a weak decreasing dependence on $A T P_{m}$ :

$J_{H, a t p}=\left(\frac{p_{13}}{p_{13}+A T P_{m}}\right)\left(\frac{p_{12}}{1+\exp \left(\left(p_{14}-\Delta \Psi\right) / p_{15}\right)}\right)$.

Graphs of the M-K (Eq. (22)) and simplified (Eq. (28)) expression are compared in Fig. 6. 
The $\mathrm{M}-\mathrm{K}$ expression for the phosphorylation rate of ADP by the ATP-synthase, $J_{F 1 F 0}$, is

$\hat{J}_{F 1 F 0}=-\rho^{F 1} \frac{M-N}{K+L}$,

where

$M=\left(100 p_{a}+p_{c 1} \mathrm{e}^{3 \Delta \Psi_{B} F R T}\right) \mathrm{e}^{A_{F 1} F R T}$,

$N=p_{a} \mathrm{e}^{3 \Delta \mu_{H} F R T}+p_{c 2} \mathrm{e}^{A_{F 1} F R T} \mathrm{e}^{3 \Delta \mu_{H} F R T}$

and $K$ and $L$ are given by Eqs. (25), (26). Because the phosphorylation rate of ADP is determined by the proton flux through the synthase, the simplified expression for $J_{F 1 F 0}$ is similar to that of $J_{H, \text { atp }}$. In fact, $J_{F 1 F 0}$ is a constant multiple of $J_{H, \text { atp }}$ :

$J_{F 1 F 0}=\left(\frac{p_{13}}{p_{13}+A T P_{m}}\right)\left(\frac{p_{16}}{1+\exp \left(\left(p_{14}-\Delta \Psi\right) / p_{15}\right)}\right)$.

The ADP phosphorylation rates computed with Eqs. (29) and (32) are shown in Fig. 7.

There is a small leak of protons across the inner mitochondrial membrane, which is larger for larger values of the electrical potential. This is linear in the $\mathrm{M}-\mathrm{K}$ model:

$\hat{J}_{H, \text { leak }}=g_{H} \Delta \mu_{H}$,

where $g_{H}=2 \times 10^{-3} \mu \mathrm{M} \mathrm{ms}^{-1} \mathrm{mV}^{-1}$. In our model we use the linear function

$J_{H, \text { leak }}=p_{17} \Delta \Psi+p_{18}$.

Note that we have replaced the $\mathrm{pH}$ dependence with a constant factor $p_{18}$. The fluxes computed using Eqs. (33) and (34) are shown in Fig. 8.

The ATP produced in the mitochondria is transported to the cytosol through the adenine nucleotide translocator. This is a carrier that exchanges one molecule of mitochondrial $\mathrm{ATP}^{4-}$ for one molecule of cytosolic $\mathrm{ADP}^{3-}$. It tends to keep the ATP/ADP ratio in the cytosol equal to that in the mitochondria. In the $\mathrm{M}-\mathrm{K}$ formulation the flux rate,
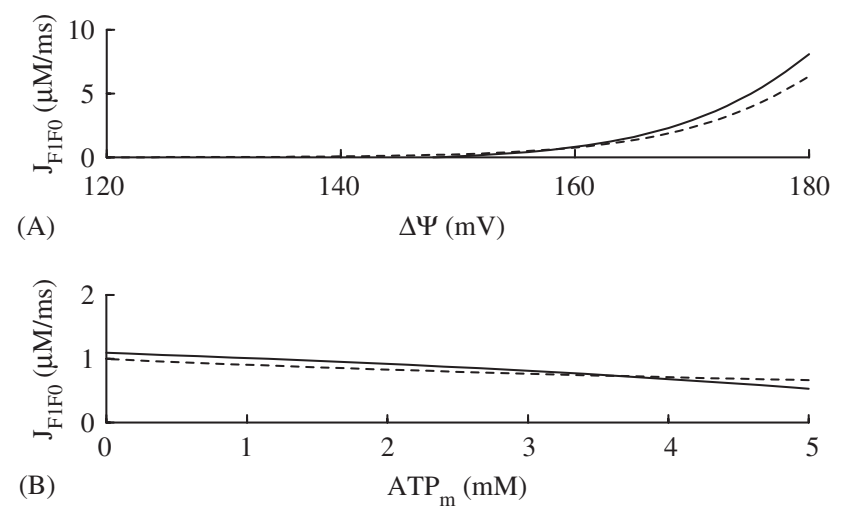

Fig. 7. Phosphorylation rate of the ATP synthase, calculated with the M-K expression (Eq. (29), solid curves) and with the simplified expression (Eq. (32), dashed curves). (A) Phosphorylation is powered by the electrical gradient, and thus increases with $\Delta \Psi$. $\left(A T P_{m}=3 \mathrm{mM}\right)$. (B) $J_{F 1 F 0}$ declines as the concentration of the reaction product is increased. $(\Delta \Psi=160 \mathrm{mV})$.

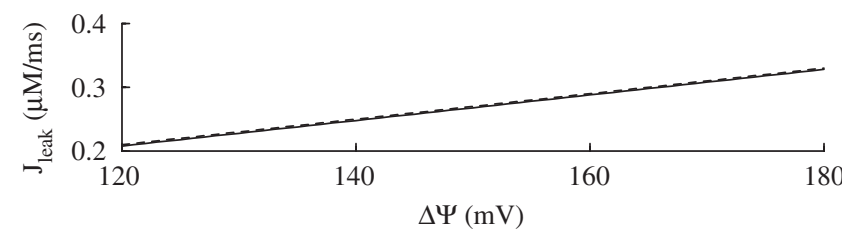

Fig. 8. Proton leakage across the inner membrane increases with the electrical potential. Computed with Eq. (33) (solid curves), and with Eq. (34) (dashed curves).
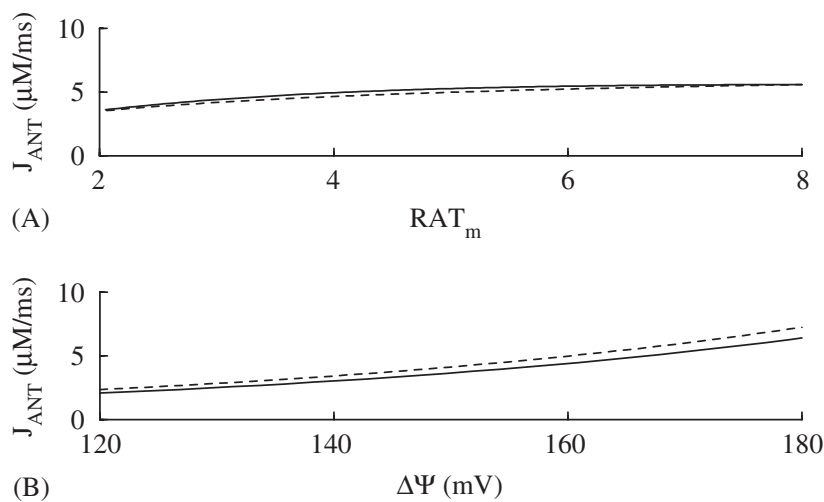

Fig. 9. Flux through the adenine nucleotide translocator, calculated with the M-K expression (Eq. (35), solid curves) and the simplified expression (Eq. (38), dashed curves). (A) Flux increases with the ATP concentration, and thus with the ATP/ADP ratio. $(\Delta \Psi=160 \mathrm{mV})$. (B) Flux increases with the inner membrane potential $\left(\mathrm{RAT}_{m}=5\right)$.

$J_{A N T}$, is

$\hat{J}_{A N T}=V_{\max A N T} \frac{R A T_{m}-0.8 R A T_{c}}{\left(1+0.11 R A T_{c}\right)\left(R A T_{m}+7.2\right) \mathrm{e}^{(-0.5 F R T \Delta \Psi)}}$,

where $V_{\max A N T}=5 \mu \mathrm{Mms}^{-1}$ is the maximum flux rate, and $R A T_{m}=\frac{A T P_{m}}{A D P_{m}}$,

$R A T_{c}=\frac{A T P_{c}}{A D P_{c}}$,

are the nucleotide ratios in the mitochondria and the cytosol, respectively. Fig. 9 shows the dependence of $J_{A N T}$ on the mitochondrial nucleotide ratio and on the inner membrane potential. Since the transporter maintains $R A T_{c} \approx R A T_{m}$, we assume that $R A T_{c}=R A T_{m}$ in the figure. The transporter is driven at a higher rate when the mitochondrial ATP/ADP ratio is larger (Fig. 9(A)), and the transport rate increases with the inner membrane potential (Fig. 9(B)). In the simplified expression, we set $R A T_{c}=R A T_{m}$ and replace the dependence on $R A T_{m}$ with a simpler functional form:

$J_{A N T}=p_{19}\left(\frac{R A T_{m}}{R A T_{m}+p_{20}}\right) \mathrm{e}^{0.5 F R T \Delta \Psi}$.

The $\mathrm{Ca}^{2+}$ uniporter carries $\mathrm{Ca}^{2+}$ from the cytosol into the mitochondrion and depends on the electrical driving 
force $\Delta \Psi$. This is described in the $\mathrm{M}-\mathrm{K}$ model by

$\hat{J}_{u n i}=V_{\max U n i}\left(\frac{O}{P+Q}\right)$,

where

$O=2 F R T\left(\Delta \Psi_{m}-\Delta \Psi^{o}\right) \frac{C a_{c}}{K_{\text {trans }}}\left(1+\frac{C a_{c}}{K_{\text {trans }}}\right)^{3}$,

$P=\left(1+\frac{C a_{c}}{K_{\text {trans }}}\right)^{4}$

$Q=\frac{L_{1}\left(1-\mathrm{e}^{-2 F R T\left(\Delta \Psi_{m}-\Delta \Psi^{o}\right)}\right)}{\left(1+C a_{c} / K_{a c t}\right)^{n_{a}}}$.

The maximum transport rate is $V_{\max U n i}=10 \mu \mathrm{Mms}^{-1}$, and $K_{\text {trans }}=19 \mu \mathrm{M}, K_{a c t}=0.38 \mu \mathrm{M}, L=110, \Delta \Psi^{o}=91 \mathrm{mV}$ is the offset membrane potential, and $C a_{c}$ is the cytosolic $\mathrm{Ca}^{2+}$ concentration. The uniporter flux is greatly simplified with the expression

$J_{\text {uni }}=\left(p_{21} \Delta \Psi-p_{22}\right) C a_{c}^{2}$.

The original and simplified expressions for the uniporter rate are shown in Fig. 10. The rate increases when either the inner membrane potential or the cytosolic $\mathrm{Ca}^{2+}$ concentration increase.

The final flux term is for the $\mathrm{Na}^{+} / \mathrm{Ca}^{2+}$ exchanger, which transports $\mathrm{Ca}^{2+}$ out of the mitochondria while bringing $\mathrm{Na}^{+}$in. The original $\mathrm{M}-\mathrm{K}$ expression for $\hat{J}_{\mathrm{NaCa}}$ was modified by Cortessa et al. to include a dependence on the mitochondrial $\mathrm{Ca}^{2+}$ concentration. The reformulated expression for $\hat{J}_{\mathrm{NaCa}}$ is

$\hat{J}_{N a C a}=V_{\text {maxNaCa}} \frac{\left(C a_{m} / C a_{c}\right) \mathrm{e}^{b F R T\left(\Delta \Psi-\Delta \Psi^{o}\right)}}{\left(1+K_{N a} / N a_{c}\right)^{3}\left(1+K_{C a} / C a_{m}\right)}$,

where $N a_{c}=10 \mathrm{mM}$ is the $\mathrm{Na}^{+}$concentration in the cytosol, $V_{\operatorname{maxNaCa}}=0.06 \mu \mathrm{Mms}^{-1}, b=0.5, K_{N a}=9.4 \mathrm{mM}$, and $K_{C a}=3.75 \times 10^{-4} \mathrm{mM}$. The simplified expression retains

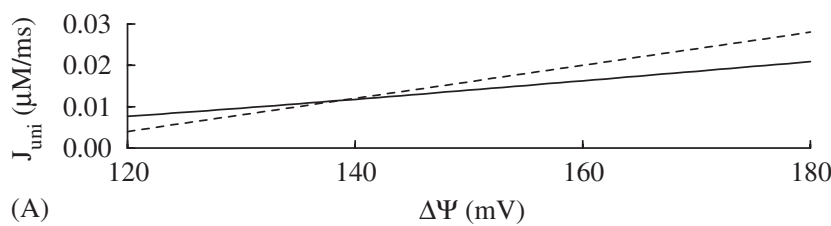

(A)

$\Delta \Psi(\mathrm{mV})$

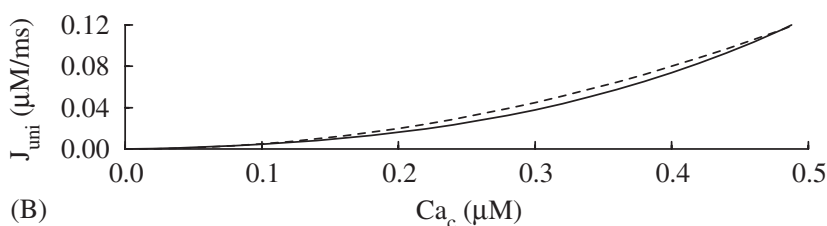

(B)

$$
\mathrm{Ca}_{\mathrm{c}}(\mu \mathrm{M})
$$

Fig. 10. The uniporter transport rate, calculated with the M-K expression (Eq. (39), solid curves) and the simplified expression (Eq. (43), dashed curves). (A) The transport rate increases with the inner membrane potential $\left(\mathrm{Ca}_{c}=0.2 \mu \mathrm{M}\right)$, and (B) with the cytosolic $\mathrm{Ca}^{2+}$ concentration $(\Delta \Psi=160 \mathrm{mV})$.
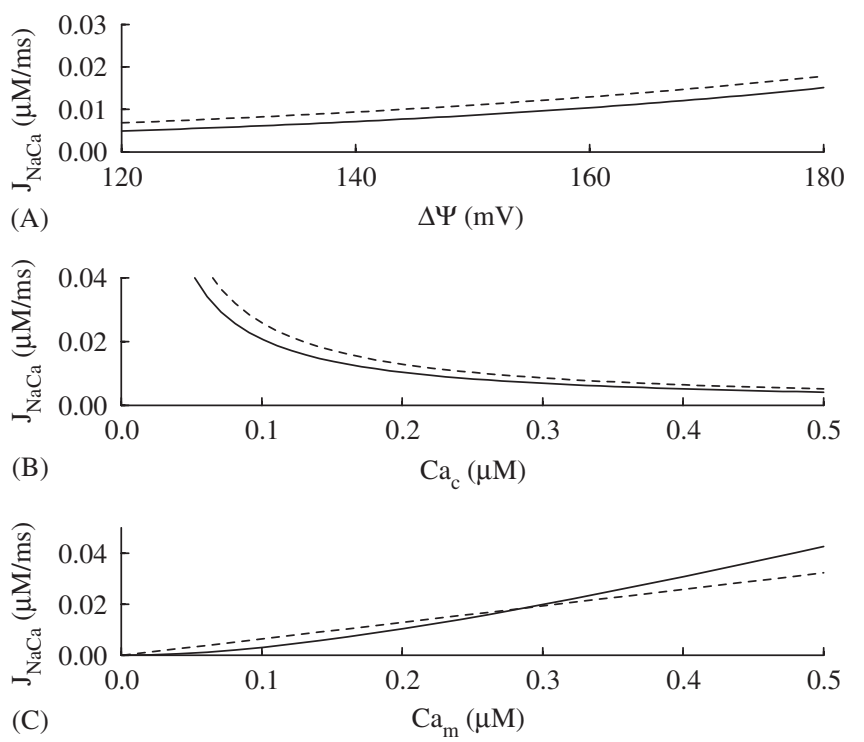

Fig. 11. The $\mathrm{Na}^{+} / \mathrm{Ca}^{2+}$ exchanger flux, calculated with the $\mathrm{M}-\mathrm{K}$ expression (Eq. (44), solid curves) and the simplified expression (Eq. (45), dashed curves). (A) The flux has a positive dependence on the inner membrane potential. $\left(C a_{c}=0.2 \mu \mathrm{M}, C a_{m}=0.2 \mu \mathrm{M}\right)$. (B) $J_{\mathrm{NaCa}}$ decreases with the cytosolic $\mathrm{Ca}^{2+}$ concentration $\left(\mathrm{Ca}_{m}=0.2 \mu \mathrm{M}\right.$, $\Delta \Psi=160 \mathrm{mV})$, and increases with the mitochondrial $\mathrm{Ca}^{2+}$ concentration $\left(C a_{c}=0.2 \mu \mathrm{M}, \Delta \Psi=160 \mathrm{mV}\right)$.

the dependence on $C a_{c}, C a_{m}$, and $\Delta \Psi$ :

$J_{N a C a}=p_{23}\left(\frac{C a_{m}}{C a_{c}}\right) \mathrm{e}^{p_{24} \Delta \Psi}$.

Fig. 11 shows $J_{\mathrm{NaCa}}$ over a range of values of its three variables. It is assumed that the cytosolic $\mathrm{Na}^{+}$concentration is constant. There is a positive dependence on the inner membrane potential (Fig. 11(A)). As expected from the nature of the $\mathrm{Na}^{+} / \mathrm{Ca}^{2+}$ exchanger, the flux is greater when the cytosolic $\mathrm{Ca}^{2+}$ concentration is lower (Fig. 11(B)), or when the mitochondrial $\mathrm{Ca}^{2+}$ concentration is greater (Fig. 11(C)).

The differential equations for the full model were solved numerically using the CVODE solver with tolerance of $10^{-9}$, implemented in the software package XPPAUT (Ermentrout, 2002). The XPPAUT software is free and can be downloaded from www.pitt.edu/ phase. The file containing the mitochondrial model can be downloaded from www.math.fsu.edu/ bertram.

\section{Mitochondrial responses to input}

An increase in the glucose concentration increases the glycolytic flux, leading to an increase in the FBP concentration. In addition, glycolysis has been shown to be oscillatory in some cases (Tornheim and Lowenstein, 1974; Longo et al., 1991), resulting in periodic pulses of FBP. We next examine the affect that a single FBP pulse has on the mitochondrial variables.

Fig. 12 shows the responses to steps in the FBP concentration to 5,10 , or $15 \mu \mathrm{M}$ (Fig. 12(A)), with the 

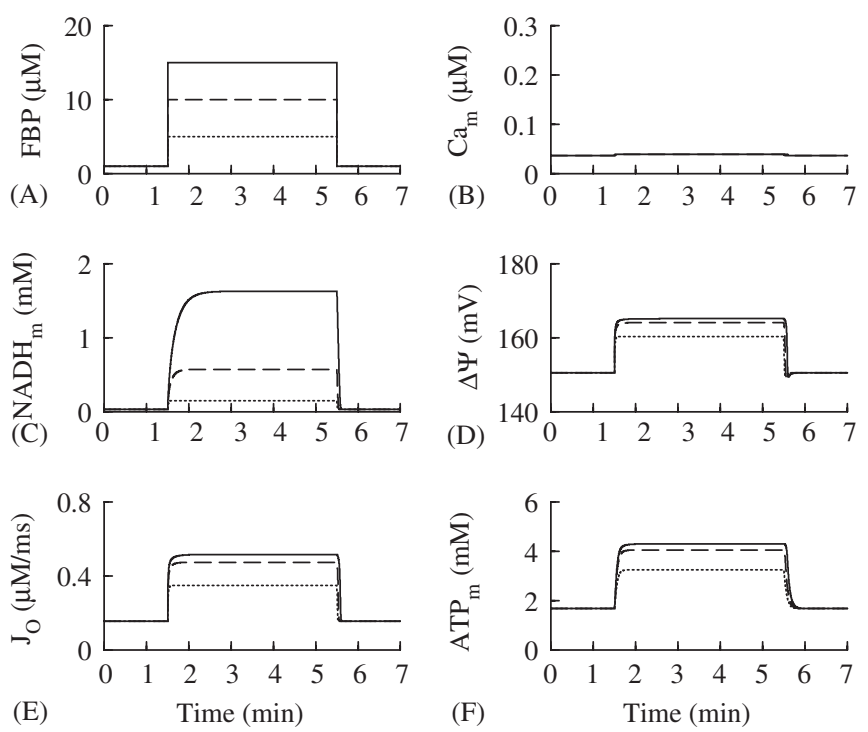

Fig. 12. The effects of FBP steps on the mitochondrial variables. The cytosolic $\mathrm{Ca}^{2+}$ concentration is $0.1 \mu \mathrm{M}$.

cytosolic $\mathrm{Ca}^{2+}$ concentration held constant at $0.1 \mu \mathrm{M}$. Each step provides fuel for the mitochondria, so there is a resulting increase in $N A D H_{m}$ (Fig. 12(C)). The elevated $N A D H_{m}$ increases respiration, increasing the oxygen consumption (Fig. 12(E)). The increased respiration hyperpolarizes the inner membrane (Fig. 12(D)), resulting in increased ATP production through the $\mathrm{F}_{1} \mathrm{~F}_{0}$ ATP synthase (Fig. 12(F)). The hyperpolarized membrane also increases the $\mathrm{Ca}^{2+}$ uniporter flux rate (Fig. 10), leading to a slight increase in the mitochondrial $\mathrm{Ca}^{2+}$ concentration (Fig. 12(B)). Since respiration saturates at large NADH concentrations (Fig. 5), reflecting metabolic control, there is a saturation of $J_{o}, \Delta \Psi$, and $A T P_{m}$ for the higher FBP concentrations.

Calcium concentration changes in the cytosol typically occur as the result of ion channel openings or $\mathrm{Ca}^{2+}$ release from the endoplasmic reticulum. In many cases, $C a_{c}$ is elevated due to bursts of action potentials. In Fig. 13 we show the response of the model to three 30-s $C a_{c}$ pulses, with FBP concentration held constant at $5 \mu \mathrm{M}$. Each pulse could reflect a burst of action potentials, which is typically tens of seconds in duration in pancreatic $\beta$-cells (Zhang et al., 2003).

The entry of $\mathrm{Ca}^{2+}$ across the inner mitochondrial membrane affects the mitochondria in two ways: the flux of positive ions depolarizes the inner membrane (the electrical effect), and the increase in $C a_{m}$ activates $\mathrm{PDH}$, increasing $N A D H_{m}$ and leading to hyperpolarization of the membrane (the dehydrogenase effect). In Fig. 13 we show the results of these combined actions (solid curves), as well as the electrical effect in isolation (dashed curves), obtained by setting $p_{3}=0$, which removes the dehydrogenase effect. Panel $\mathrm{C}$ shows that the $\mathrm{Ca}^{2+}$ pulses increase $N A D H_{m}$ (solid curve), as expected from the dehydrogenase effect. This transiently enhances respiration, as shown by
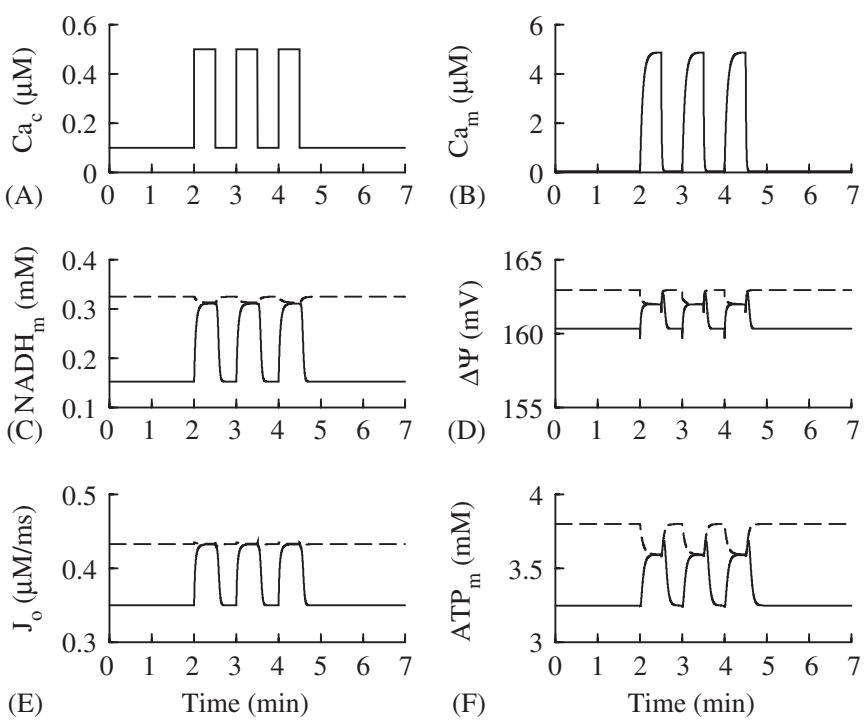

Fig. 13. The effects of $\mathrm{Ca}^{2+}$ steps on the mitochondrial variables. The dashed curves show the result when the stimulatory effect of $\mathrm{Ca}^{2+}$ on $\mathrm{PDH}$ activity is saturated by setting $p_{3}=0$ in Eq. (10). The FBP concentration is $5 \mu \mathrm{M}$.

increases in the oxygen consumption (Fig. 13(E)). The enhanced respiration hyperpolarizes the inner membrane (Fig. 13(D)), increasing the ATP production (Fig. 13(F)). Thus, the $\mathrm{Ca}^{2+}$ pulses produce upward "teeth" in the mitochondrial variables and the $\mathrm{O}_{2}$ flux. However, when $p_{3}=0$, isolating the electrical effect, the $\mathrm{Ca}^{2+}$ pulses transiently depolarize the inner membrane (Fig. 13(D), dashed) and reduce ATP production (Fig. 13(F), dashed). Thus, the teeth in the mitochondrial variables are reversed when the dehydrogenase effect is removed, and measurements of $\Delta \Psi$ or $A T P_{m}$ in response to $\mathrm{Ca}^{2+}$ pulses could be used to determine which of the two competing effects of $\mathrm{Ca}^{2+}$ flux dominates within the mitochondria.

The direction of the teeth in $\Delta \Psi$ and $A T P_{m}$ can also be flipped from up to down by increasing the flux rate of the $\mathrm{Ca}^{2+}$ uniporter $J_{\text {uni }}$. In Fig. 14 we show the response of the system to $\mathrm{Ca}^{2+}$ pulses with the default parameter values (solid curves, the control) and with the uniporter flux parameter $p_{21}$ increased from 0.01 to $0.02 \mu \mathrm{M}^{-1} \mathrm{~ms}^{-1} \mathrm{mV}^{-1}$ (dashed curves). This two-fold increase in the parameter value results in an approximately four-fold increase in $C a_{m}$ during the sequence of $C a_{c}$ pulses (Fig. 14(B)). The basal level of $C a_{m}$ is also elevated, so that the basal level of $N A D H_{m}$ is higher than the control. In fact, the stimulatory effect of $\mathrm{Ca}^{2+}$ on PDH is nearly saturated even at the basal level of $C a_{m}$, so the size of the upward $N A D H_{m}$ teeth is significantly less than the control (Fig. 14(C)). This is reflected in the oxygen consumption (Fig. 14(E)), since the smaller $N A D H_{m}$ teeth stimulate less respiration. Along with the blunted stimulatory effect of $\mathrm{Ca}^{2+}$ on PDH, there is now greater $\mathrm{Ca}^{2+}$ flux across the membrane during the $\mathrm{Ca}^{2+}$ pulses. Hence, the depolarizing electrical effect of $\mathrm{Ca}^{2+}$ is enhanced while the hyperpolarizing dehydrogenase effect is reduced. The net effect is that the $\Delta \Psi$ teeth are 
now downward (depolarizing) rather than upward (Fig. 14(D)). Consequently, the $A T P_{m}$ teeth are also downward (Fig. 14(F)). In summary, any maneuover that saturates the stimulatory effect of $\mathrm{Ca}^{2+}$ on $\mathrm{PDH}$ while increasing $\mathrm{Ca}^{2+}$ influx across the inner membrane has the potential of reversing the $\Delta \Psi$ and $A T P_{m}$ teeth from upward to downward.

In a recent in vitro experimental study of metabolism in pancreatic islets, the $\mathrm{NAD}(\mathrm{P}) \mathrm{H}$ level was measured simultaneously with the cytosolic $\mathrm{Ca}^{2+}$ concentration
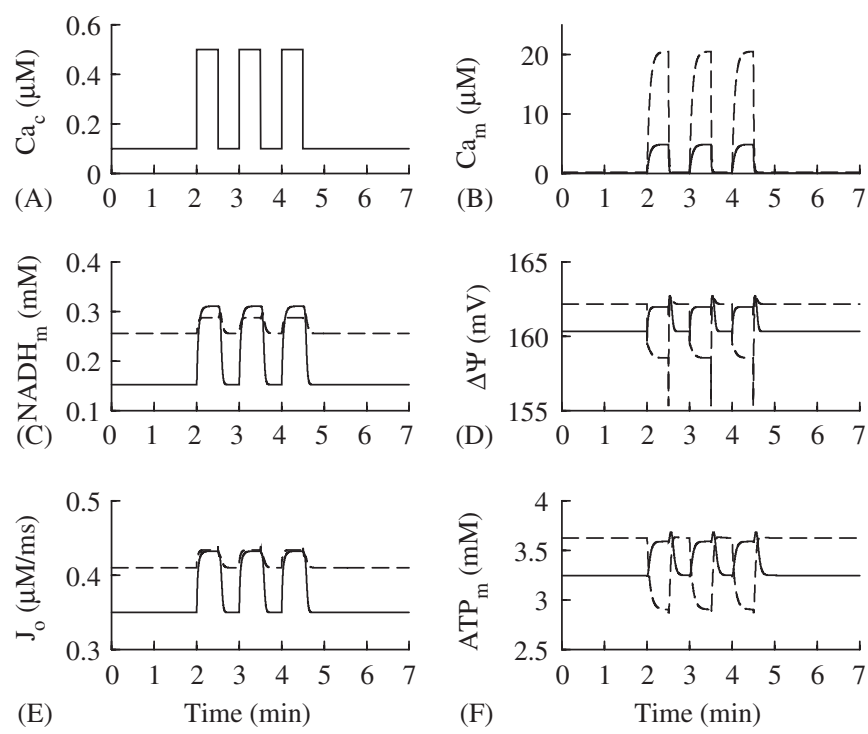

Fig. 14. The effects of $\mathrm{Ca}^{2+}$ steps on the mitochondrial variables. In one simulation the uniporter flux is doubled $\left(p_{21}=0.02 \mu \mathrm{M}^{-1} \mathrm{~ms}^{-1} \mathrm{mV}^{-1}\right.$, dashed curves). This reverses the direction of the $\Delta \Psi$ and $A T P_{m}$ teeth. The FBP concentration is $5 \mu \mathrm{M}$.
(Luciani et al., 2006). (NAD(P)H is the sum of the NADH and NADPH concentrations.) When islets were maintained in a low glucose concentration $(3 \mathrm{mM})$ and then depolarized by adding $30 \mathrm{mM} \mathrm{KCl}$ to the bath, both the $\mathrm{Ca}^{2+}$ concentration and the $\mathrm{NAD}(\mathrm{P}) \mathrm{H}$ concentration (measured through autofluorescence) increased. An example is shown in Fig. 15(A). The experiment was repeated in islets maintained in a high glucose concentration $(10 \mathrm{mM})$. At this level of glucose the islet $\mathrm{Ca}^{2+}$ concentration oscillates due to bursting electrical activity, so the pharmacological agent diazoxide was added to the bath to hyperpolarize the islets and thus bring $C a_{c}$ to a steady low level. When the islets were then depolarized with $30 \mathrm{mM} \mathrm{KCl}$ the $\mathrm{Ca}^{2+}$ concentration increased as expected. However, the $\mathrm{NAD}(\mathrm{P}) \mathrm{H}$ concentration decreased (Fig. 15(B)). Thus, an increase in the cytosolic $\mathrm{Ca}^{2+}$ concentration elevates the $\mathrm{NAD}(\mathrm{P}) \mathrm{H}$ concentration in low glucose, but lowers it in high glucose.

The small decreases in $N A D H_{m}$ during $\mathrm{Ca}^{2+}$ pulses in Fig. 13(C) in the case where the dehydrogenase effect was removed suggests that the model may be able to explain the results of Fig. 15. That is, if the electrical effect of $\mathrm{Ca}^{2+}$ dominates the dehydrogenase effect, then an increase in $C a_{m}$ should be able to produce downward $N A D H_{m}$ teeth, consistent with Fig. 15(B). To accentuate the electrical effect we used larger pulses of $\mathrm{Ca}_{c}$ than in previous simulations (pulsing from 0.1 to $2 \mu \mathrm{M}$ ). From the $N A D H_{m}$ differential equation (Eq. (1)), we see that $N A D H_{m}$ can decline only if the change in $J_{o}$ is greater than the change in $J_{P D H}$ during the $\mathrm{Ca}^{2+}$ pulses. Fig. 16(E) shows that in the low glucose simulation $(\mathrm{FBP}=1 \mu \mathrm{M})$, the downward $\Delta \Psi$ teeth have little effect on $J_{o}$; a depolarization of $20 \mathrm{mV}$ from $\approx 152 \mathrm{mV}$ (open circle) to
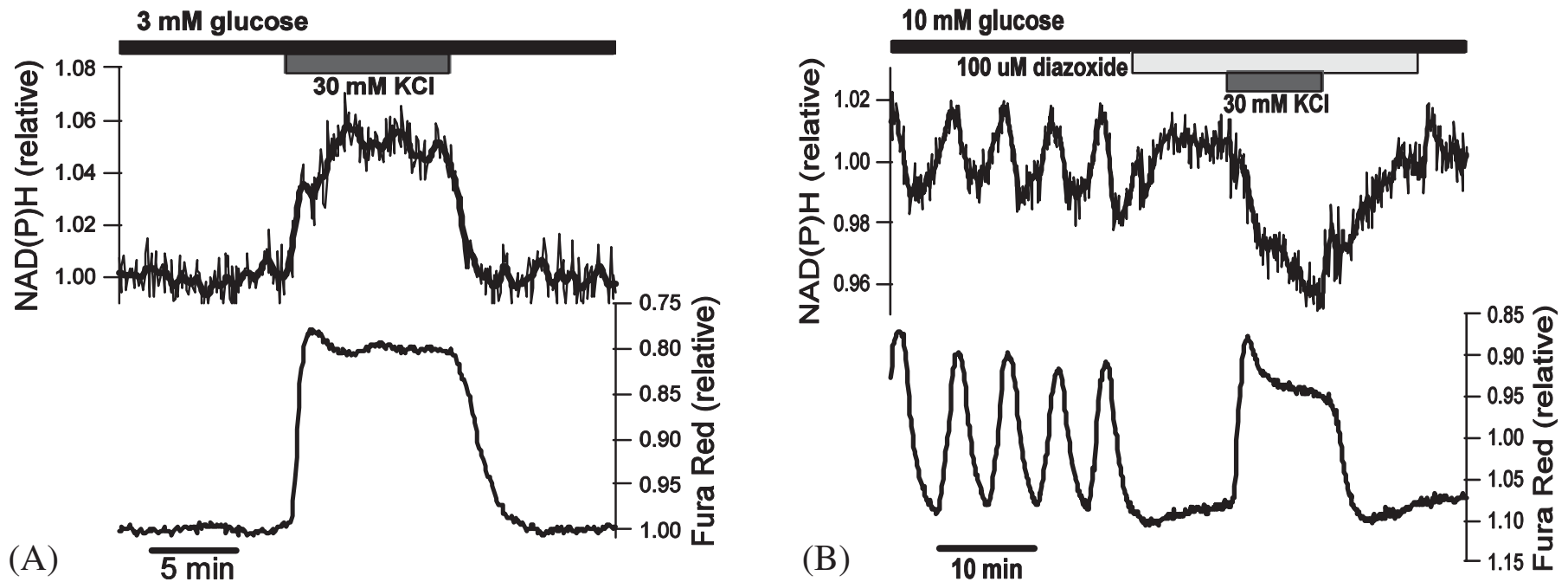

Fig. 15. Experimental simultaneous measurements of $\mathrm{NAD}(\mathrm{P}) \mathrm{H}$ autofluorescence (top) and cytosolic $\mathrm{Ca}^{2+}$ concentration (bottom) in pancreatic islets. These data are similar to those reported in an earlier study (Luciani et al., 2006). (A) When the islet was maintained in a low glucose concentration ( $3 \mathrm{mM}$ ), depolarization with $30 \mathrm{mM} \mathrm{KCl}$ increased the cytosolic $\mathrm{Ca}^{2+}$ concentration and the $\mathrm{NAD}(\mathrm{P}) \mathrm{H}$ autofluorescence (indicative of an increase in concentration). (B) When the islet was maintained in a high glucose concentration $(10 \mathrm{mM})$ and the $\mathrm{Ca}^{2+}$ oscillations were abolished with the hyperpolarizing agent diazoxide, subsequent depolarization with $30 \mathrm{mM} \mathrm{KCl}$ increased the cytosolic $\mathrm{Ca}^{2+}$ concentration, but decreased the $\mathrm{NAD}(\mathrm{P}) \mathrm{H}$ autofluorescence. 

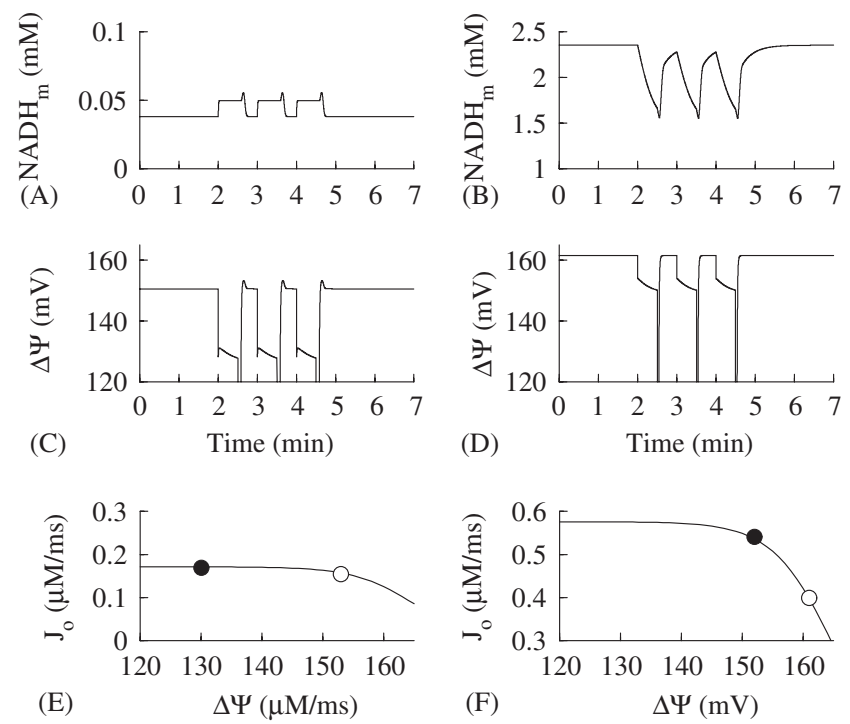

Fig. 16. Model illustration of how the $N A D H_{m}$ teeth can be upward in low glucose ( $\mathrm{FBP}=1 \mu \mathrm{M}$, left column) and downward in high glucose (FBP $=10 \mu \mathrm{M}$, right column). Large pulses of $C a_{c}$ are used $\left(C a_{c}\right.$ is pulsed from 0.1 to $2 \mu \mathrm{M}$ ) to accentuate the electrical effect. The bottom panels show the $J_{o}$ vs. $\Delta \Psi$ curve (as in Fig. $4(\mathrm{~A})$ ) at the $N A D H_{m}$ levels corresponding to low (panel E) and high (panel F) glucose. (The curve has been shifted to the left in both cases by setting $p_{6}=p_{10}=165 \mathrm{mV}$.) The open circle represents the values of $\Delta \Psi$ and $J_{o}$ prior to a $\mathrm{Ca}^{2+}$ pulse. The closed circle represents the values during a $\mathrm{Ca}^{2+}$ pulse. The change in $J_{o}$ during a pulse is much greater in high glucose than in low glucose, causing the $N A D H_{m}$ teeth to be downward in high glucose (panel B) and upward in low glucose (panel A).

$\approx 130 \mathrm{mV}$ (filled circle) results in almost no change in $J_{o}$. In fact, the change in $J_{P D H}$ is larger, so the $N A D H_{m}$ teeth are upward (Fig. 16(A)). In contrast, in the high glucose simulation $(\mathrm{FBP}=10 \mu \mathrm{M})$, the downward $\Delta \Psi$ teeth cause a significantly greater increase in $J_{o}$ (Fig. 16F); a depolarization of less than $10 \mathrm{mV}$ increases $J_{o}$ by approximately $50 \%$. Thus, the change in $J_{o}$ is greater than the change in $J_{P D H}$ during a $\mathrm{Ca}^{2+}$ pulse in high glucose. As a result, the $N A D H_{m}$ teeth are downward (Fig. 16(B)). Therefore, the model is able to account for the experimental data in Fig. 15. The model is also consistent with the finding that the $\mathrm{Ca}^{2+}$-induced decline in $\mathrm{NAD}(\mathrm{P}) \mathrm{H}$ in high glucose is converted to an increase in $\mathrm{NAD}(\mathrm{P}) \mathrm{H}$ when the ETC is blocked (Luciani et al., 2006). Blocking the ETC depolarizes the mitochondrial inner membrane and reduces the magnitude of the $\mathrm{Ca}^{2+}$-induced increase in $J_{o}$.

Given the complexity of mitochondrial metabolism, it is hard to predict the effects of genetic mutations in mitochondrial enzymes without a mathematical model. The current model facilitates such predictions. As an example, we consider the nuclear-encoded mitochondrial enzyme nicotinamide nucleotide transhydrogenase (Nnt). This enzyme detoxifies reactive oxygen species (ROS) which, when elevated, can reduce mitochondrial ATP production (Hoek and Rydstrom, 1988). The accumulation of ROS increases the activity of uncoupling protein 2 , resulting in enhanced proton leakage across the inner
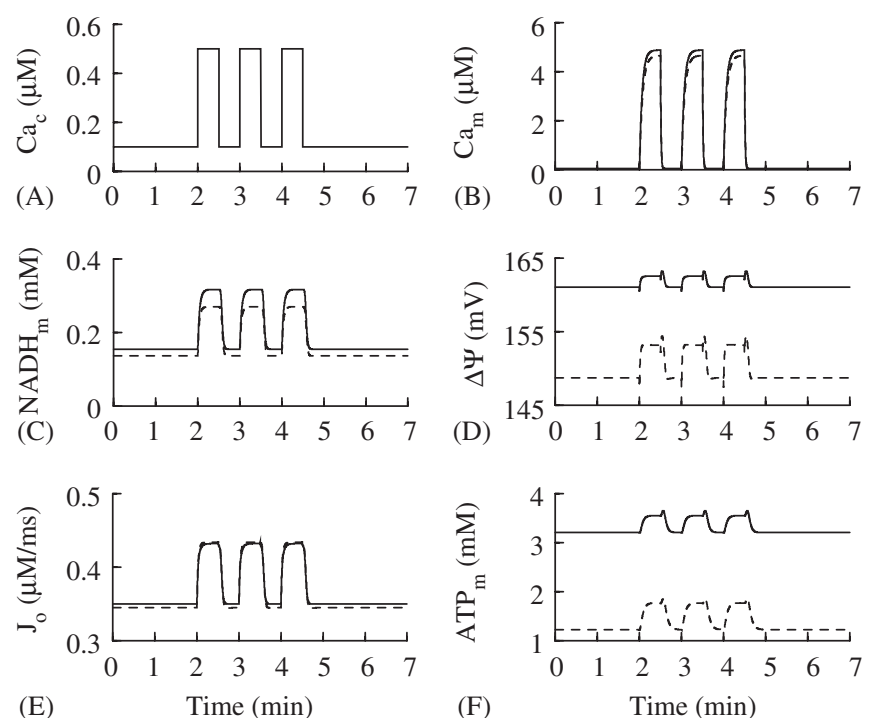

Fig. 17. In the Nnt mutant, proton leakage across the inner mitochondrial membrane is increased. This is simulated by increasing $p_{17}$ from $0.002 \mu \mathrm{Mms}^{-1} \mathrm{mV}^{-1}$ (wild type, solid curves) to $0.02 \mu \mathrm{Mms}^{-1} \mathrm{mV}^{-1}$ (mutant, dashed curves). This lowers the membrane potential and basal level of ATP, but also increases the size of the teeth in $\Delta \Psi$ and $A T P_{m}$. The FBP concentration is $5 \mu \mathrm{M}$.

membrane (Remedi et al., 2006). Thus, mutations in Nnt that diminish its activity will likely lead to an increase in proton leakage. Recent evidence suggests that diminished Nnt activity in mouse mutants results in reduced insulin release from pancreatic $\beta$-cells (Freeman et al., 2006), possibly due to depolarization of $\beta$-cell mitochondria and subsequent reduction in ATP production that results from increased proton leakage.

How would a mutation in Nnt affect the $\mathrm{Ca}^{2+}$ response of the mitochondrial variables? To simulate a negative Nnt mutation we increased the proton leak rate tenfold by increasing $p_{17}$ from 0.002 to $0.02 \mu \mathrm{Mms}^{-1} \mathrm{mV}^{-1}$. As expected, one effect is to reduce the inner membrane potential, and thus the mitochondrial ATP concentration. What is not so obvious is that the Nnt mutation also increases the size of the teeth in $\Delta \Psi$ and $A T P_{m}$ (Fig. 17). Thus, the effect of $\mathrm{Ca}^{2+}$ feedback onto metabolism is amplified in this mutation. This effect, which is due to metabolic control, would likely have been overlooked without a mathematical model.

\section{Discussion}

The simplified model for mitochondrial ATP production that we have described has several advantages over the original $\mathrm{M}-\mathrm{K}$ or Cortassa et al. models upon which it was based. The relative simplicity of the model makes it possible to readily determine the effects of the mitochondrial input on each of the flux and reaction terms. This facilitates comprehension of the model. It is also clear from the new model how changes in parameter values will affect the flux and reaction terms. This helped considerably when 
trying to reproduce the reversal of the $N A D H_{m}$ teeth in low vs. high glucose (Figs. 15 and 16). With the original M-K model it is unlikely that we would have realized how this could occur. Also, the simplicity of the model facilitates model studies of mitochondrial gene mutations, and the effects of pharmacological agents that target the mitochondria. Finally, the simplified model may be preferable to more complex models when it forms only a portion of a larger cellular model. Indeed, this was part of our motivation, since our long-term goal is to incorporate all stages of glucose metabolism into a model of metabolic and calcium oscillations in the pancreatic $\beta$-cell.

Our model simulations focused largely on the response of the mitochondrial variables to pulses of $\mathrm{Ca}^{2+}$. Our interest in this is driven by several studies in which mitochondrial variables (or oxygen consumption) were measured in pancreatic islets. In one study, the mitochondrial membrane potential was measured with rhodamine 123 simultaneously with the cytosolic $\mathrm{Ca}^{2+}$ concentration, which was measured using fura-2/AM (Krippeit-Drews et al., 2000). During glucose stimulation a series of $C a_{c}$ pulses were observed, corresponding to bursts of action potentials. Teeth in $\Delta \Psi$ were clearly evident, with each $\Delta \Psi$ deflection correlated with a $C a_{c}$ pulse. In this case, the $\Delta \Psi$ teeth were downward, suggesting that the electrical effect of $\mathrm{Ca}^{2+}$ on the mitochondria dominated the dehydrogenase effect. In another study in which $\mathrm{Ca}^{2+}$ and $\Delta \Psi$ were measured simultaneously, elevations in the $\mathrm{Ca}^{2+}$ concentration through various means depolarized the mitochondrial membrane when the glucose concentration was at a stimulatory level. In basal glucose, elevations in the $\mathrm{Ca}^{2+}$ concentration had the opposite effect (Kindmark et al., 2001). Again, this suggests that the teeth are upward (hyperpolarizing) when the dehydrogenase effect dominates, and downward (depolarizing) when the electrical effect dominates (e.g., when the dehydrogenase is saturated, as could occur when the glucose concentration is high). In another study (Jung et al., 2000), an oxygen microsensor was used to measure oxygen levels in mouse islets. The cytosolic $\mathrm{Ca}^{2+}$ concentration was measured simultaneously. Teeth in the oxygen consumption were observed, with each tooth (increase in oxygen consumption) corresponding to a $C a_{c}$ pulse. Each $C a_{c}$ pulse is likely due to a burst of action potentials. Oxygen teeth were again seen in a later study by the same lab (Dahlgren et al., 2005).

Finally, we point out that while our simplified mitochondrial model has many applications, the more complex models of $\mathrm{M}-\mathrm{K}$ or Cortassa et al. have the advantage that the parameters represent specific biophysical rates. Thus, one could develop improved models by obtaining more accurate measurements of the various rate constants. Our parameters, in contrast, are based on curve fitting to the model of Cortassa et al. If an improved detailed mitochondrial model were developed, one could again use a curve-fitting approach to develop a simplified model that is more physiologically accurate than ours. Indeed, there are other models of mitochondrial metabolism, each of which focuses on specific aspects of metabolism (see Jafri et al., 2001 for review). We have chosen to use the $\mathrm{M}-\mathrm{K}$ model since its development was based largely on data from pancreatic $\beta$-cells, the cell type of interest to us. Overall, we believe that both detailed and simplified mitochondrial models are useful in understanding the function of mitochondria and how they interact with other components of the cell.

\section{Acknowledgments}

R.B. was partially supported by National Science Foundation Grants DMS-0311856 and DMS-0613179. M.G.P. and D.S.L. were partially supported by the European Union through the Network of Excellence BioSim, LSHB-CT-2004-005137. A.S. was supported by the intramural research program of the National Institutes of Health, National Institute of Diabetes and Digestive and Kidney Diseases.

\section{References}

Cortassa, S., Aon, M.A., Marban, E., Winslow, R.L., O’Rourke, B., 2003. An integrated model of cardiac mitochondrial energy metabolism and calcium dynamics. Biophys. J. 84, 2734-2755.

Dahlgren, G.M., Kauri, L.M., Kennedy, R.T., 2005. Substrate effects on oscillations in metabolism, calcium and secretion in single mouse islets of Langerhans. Biochem. Biophys. Res. Commun. 1724, 23-36.

Dudycha, S., 2000. Master's Thesis, Johns Hopkins University.

Ermentrout, G.B., 2002. Simulating, Analyzing, and Animating Dynamical Systems: A Guide to XPPAUT for Researchers and Students. SIAM Books, Philadelphia.

Freeman, H., Shimomura, K., Horner, E., Cox, R.D., Ashcroft, F.M., 2006. Nicotinamide nucleotide transhydrogenase: a key role in insulin secretion. Cell Metab. 3, 35-45.

Hoek, J.B., Rydstrom, J., 1988. Physiological roles of nicotinamide nucleotide transhydrogenase. Biochem. J. 254, 1-10.

Jafri, M., Dudycha, S.J., O'Rourke, B., 2001. Cardiac energy metabolism: models of cellular respiration. Annu. Rev. Biomed. Eng. 3, 57-81.

Jung, S.-K., Kauri, L.M., Qian, W.-J., Kennedy, R.T., 2000. Correlated oscillations in glucose consumption oxygen consumption, and intracellular free $\mathrm{Ca}^{2+}$ in single islets of Langerhans. J. Biol. Chem. $275,6642-6650$.

Kindmark, H., Köhler, M., Brown, G., Bränström, R., Larsson, O., Berggren, P.-O., 2001. Glucose-induced oscillations in cytoplasmic free $\mathrm{Ca}^{2+}$ concentration precede oscillations in mitochondrial membrane potential in the pancreatic $\beta$-cell. J. Biol. Chem. 276, 34530-34536.

Krippeit-Drews, P., Düfer, M., Drews, G., 2000. Parallel oscillations of intracellular calcium activity and mitochondrial membrane potential in mouse pancreatic $\beta$-cells. Biochem. Biophys. Res. Commun. 267, $179-183$.

Longo, E.A., Tornheim, K., Deeney, J.T., Varnum, B.A., Tillotson, D., Prentki, M., Corkey, B.E., 1991. Oscillations in cytosolic free $\mathrm{Ca}^{2+}$, oxygen consumption, and insulin secretion in glucose-stimulated rat pancreatic islets. J. Biol. Chem. 266, 9314-9319.

Luciani, D.S., Misler, S., Polonsky, K.S., 2006. $\mathrm{Ca}^{2+}$ controls slow NAD $(\mathrm{P}) \mathrm{H}$ oscillations in glucose-stimulated mouse pancreatic islets. J. Physiol. 572, 379-392.

Magnus, G., Keizer, J., 1997. Minimal model of $\beta$-cell mitochondrial $\mathrm{Ca}^{2+}$ handling. Am. J. Physiol. 273, C717-C733.

Magnus, G., Keizer, J., 1998a. Model of $\beta$-cell mitochondrial calcium handling and electrical activity. I. Cytoplasmic variables. Am. J. Physiol. 274, C1158-C1173. 
Magnus, G., Keizer, J., 1998b. Model of $\beta$-cell mitochondrial calcium handling and electrical activity. II. Mitochondrial variables. Am. J. Physiol. 274, C1174-C1184.

Remedi, M.S., Nichols, C.G., Koster, J.C., 2006. The mitochondria and insulin release: Nnt just a passing relationship. Cell Metab. 3, 5-7.

Tornheim, K., 1979. Oscillations of the glycolytic pathway and the purine nucleotide cycle. J. Theor. Biol. 79, 491-541.

Tornheim, K., Lowenstein, J.M., 1974. The purine nucleotide cycle. IV. Interactions with oscillations of the glycolytic pathway in muscle extracts. J. Biol. Chem. 249, 3241-3247.
Toye, A.A., Lippiat, J.D., Proks, P., Shimomura, K., Bentley, L., Hugill, A., Mijat, V., Goldsworthy, M., Moir, L., Haynes, A., Quarterman, J., Freeman, H.C., Ashcroft, F.M., Cox, R.D., 2005. A genetic and physiological study of impaired glucose homeostasis control in C57BL/6J mice. Diabetologia 48, 675-686.

Zhang, M., Goforth, P., Bertram, R., Sherman, A., Satin, L., 2003. The $\mathrm{Ca}^{2+}$ dynamics of isolated mouse $\beta$-cells and islets: Implications for mathematical models. Biophys. J. 84, 2852-2870. 